KEK-TH-1357

\title{
Tensor-polarized quark and antiquark distribution functions in a spin-one hadron
}

\author{
S. Kumano ${ }^{1}$ \\ ${ }^{1}$ KEK Theory Center, Institute of Particle and Nuclear Studies \\ High Energy Accelerator Research Organization (KEK) \\ and Department of Particle and Nuclear Studies \\ Graduate University for Advanced Studies \\ 1-1, Ooho, Tsukuba, Ibaraki, 305-0801, Japan
}

(Dated: June 14, 2010)

\begin{abstract}
To understand orbital-angular-momentum contributions is becoming crucial for clarifying nucleonspin issue in the parton level. Twist-two structure functions $b_{1}$ and $b_{2}$ for spin-one hadrons could probe orbital-angular-momentum effects, which reflect a different aspect from current studies for the spin-1/2 nucleon, since they should vanish if internal constituents are in the $S$ state. These structure functions are related to tensor structure in spin-one hadrons. Studies of such tensor structure will open a new field of high-energy spin physics. The structure functions $b_{1}$ and $b_{2}$ are described by tensor-polarized quark and antiquark distributions $\delta_{T} q$ and $\delta_{T} \bar{q}$. Using HERMES data on the $b_{1}$ structure function for the deuteron, we made an analysis of extracting the distributions $\delta_{T} q$ and $\delta_{T} \bar{q}$ in a simple $x$-dependent functional form. Optimum distributions are proposed for the tensor-polarized valence and antiquark distribution functions from the analysis. A finite tensor polarization is obtained for antiquarks if we impose a constraint that the first moments of tensorpolarized valence-quark distributions vanish. It is interesting to investigate a physics mechanism to create a finite tensor-polarized antiquark distribution.

PACS numbers: $13.60 . \mathrm{Hb}, 13.88 .+\mathrm{e}$
\end{abstract}

Origin of nucleon spin has been investigated extensively after the EMC discovery that almost none of nucleon spin is carried by quarks [1]. Recent studies of polarized parton distribution functions (PDFs) are found in Ref. 2]. Although a gluon-spin contribution is not determined accurately, orbital angular momenta are likely to be the crucial quantities in explaining the nucleon spin. Such contributions have been investigated recently by generalized parton distribution functions in lepton scattering [3] and will be studied possibly at hadron facilities [4].

There are other quantities which are sensitive to the orbital angular momenta. For example, there are twisttwo structure functions $b_{1}$ and $b_{2}$ in spin-one hadrons [5, [6]. They could be related to the orbital angular momenta of internal constituents because they vanish if the constituents are in the $S$ wave. Of course, they probe a different aspect of orbital-angular-momentum effects from the current ones for the nucleon because they are related to tensor-structure nature of spin-one hadrons. It is noteworthy that tensor structure is not understood at all in the parton level, which suggests that a new field of spin physics should be created by investigating the tensor-polarized structure functions.

New polarized structure functions $\left(b_{1}, b_{2}, b_{3}\right.$, and $\left.b_{4}\right)$ were introduced in describing lepton deep inelastic scattering from a spin-one hadron [5 7]. A useful sum rule for the twist-two function $b_{1}$ was proposed in Ref. [8], and it is partially used in this work. In conventional hadron models, such tensor structure arises due to the $D$-state admixture [5, 6, 9], pions [10], and shadowing effects [11] if the target is the deuteron. However, the tensor structure would not be simply described by such conventional models at high energies according to our experience on the nucleon-spin issue. On the other hand, a theoretical formalism was developed in Ref. [12] to investigate the tensor-polarized distributions at hadron facilities by Drell-Yan processes with polarized deuteron. There are related theoretical studies such as new fragmentation functions [13], generalized parton distributions 14], target mass corrections [15], positivity constraints [16], lattice QCD estimate [17], projection operators of $b_{1-4}$ [18] for spin-one hadrons. The first measurement of the structure function $b_{1}$ was done by the HERMES collaboration in 2005 [19].

The purpose of this research is to propose a simple parametrization for the tensor-polarized quark and antiquark distribution functions by analyzing the HERMES data. It is intended to understand the current status of the tensor distributions. Obtained distributions could be used for comparing them with theoretical model calculations and for proposing future experiments.

The structure function $b_{1}$ is defined in the hadron tensor $W_{\mu \nu}[6,18]$. It is expressed in term of tensor-polarized distributions $\left(\delta_{T} q\right.$ and $\left.\delta_{T} \bar{q}\right)$ as $[6,8,[20]$

$$
b_{1}\left(x, Q^{2}\right)=\frac{1}{2} \sum_{i} e_{i}^{2}\left[\delta_{T} q_{i}\left(x, Q^{2}\right)+\delta_{T} \bar{q}_{i}\left(x, Q^{2}\right)\right],
$$

where $i$ indicates the flavor of a quark and $e_{i}$ is the charge of the quark. The variables $Q^{2}$ and $x$ are defined by the momentum transfer $q$ as $Q^{2}=-q^{2}$ and $x=Q^{2} /\left(2 M_{N} \nu\right)$, where $M_{N}$ and $\nu$ are the nucleon mass and the energy transfer, respectively. Hereafter, the $Q^{2}$ dependence is not explicitly written in the PDFs. In this work, the $b_{1}, \delta_{T} q_{i}, \delta_{T} \bar{q}_{i}$, and unpolarized PDFs are defined by the ones per nucleon for a nuclear target, namely they are 
divided by the factor of two if it is the deuteron. The tensor-polarized distribution $\delta_{T} q$ is defined by

$$
\delta_{T} q_{i}(x) \equiv q_{i}^{0}(x)-\frac{q_{i}^{+1}(x)+q_{i}^{-1}(x)}{2},
$$

where $q_{i}^{\lambda}$ indicates an unpolarized-quark distribution in the hadron spin state $\lambda$, and it is also defined the one per nucleon. Namely, $\delta_{T} q$ indicates an unpolarized-quark distribution in a tensor-polarized spin-one hadron. It should be noted that the notation $\delta_{T} q$ is not the transversity distribution, for which similar notations $\left(\delta\right.$ or $\left.\Delta_{T}\right)$ are used in nucleon-spin studies, throughout this article. A sum rule exists for $b_{1}$ in a parton model [8]:

$$
\int d x b_{1}(x)=-\frac{5}{24} \lim _{t \rightarrow 0} t F_{Q}(t)=0,
$$

if the tensor-polarized antiquark distributions vanish $\int d x \delta_{T} \bar{q}(x)=0$. Here, $F_{Q}(t)$ is the electric quadrupole form factor of a spin-one hadron at the momentum squared $t$.

We analyze the HERMES $b_{1}$ data for the deuteron. The tensor-polarized distributions are introduced as the unpolarized PDFs in the deuteron $(D)$ multiplied by a common weight function $\delta_{T} w(x)$ :

$$
\begin{aligned}
& \delta_{T} q_{i v}^{D}(x)=\delta_{T} w(x) q_{i v}^{D}(x), \\
& \delta_{T} \bar{q}_{i}^{D}(x)=\alpha_{\bar{q}} \delta_{T} w(x) \bar{q}_{i}^{D}(x) .
\end{aligned}
$$

Namely, certain fractions of quark and antiquark distributions are tensor polarized and such probabilities are given by the function $\delta_{T} w(x)$ and an additional constant $\alpha_{\bar{q}}$ for antiquarks in comparison with the quark polarization. The $x$ dependence of $\delta_{T} w(x)$ for antiquarks could be different in general from the one for quarks. However, it is not the stage of suggesting such a difference from experimental measurements as it will become obvious later in this article.

It is known that nuclear modifications are less than a few percent for the unpolarized PDFs in the deuteron [21]. The tensor-polarized distributions cannot be determined within a few percent accuracy at this stage. Therefore, the nuclear modifications are neglected in $q_{i}^{D}$ and $\bar{q}_{i}^{D}$. Then, the PDFs in the deuteron are written by a simple addition of proton and neutron contributions: $q_{i}^{D}=\left(q_{i}^{p}+q_{i}^{n}\right) / 2$ and $\bar{q}_{i}^{D}=\left(\bar{q}_{i}^{p}+\bar{q}_{i}^{n}\right) / 2$. Furthermore, isospin symmetry is assumed for relating the PDFs of the neutron to the ones of the proton: $u_{n}=d, d_{n}=u$, $\bar{u}_{n}=\bar{d}$, and $\bar{d}_{n}=\bar{u}$. Then, the tensor polarized distributions are

$$
\begin{aligned}
\delta_{T} q_{v}^{D}(x) & \equiv \delta_{T} u_{v}^{D}(x)=\delta_{T} d_{v}^{D}(x) \\
& =\delta_{T} w(x) \frac{u_{v}(x)+d_{v}(x)}{2}, \\
\delta_{T} \bar{q}^{D}(x) & \equiv \delta_{T} \bar{u}^{D}(x)=\delta_{T} \bar{d}^{D}(x)=\delta_{T} s^{D}(x)=\delta_{T} \bar{s}^{D}(x) \\
& =\alpha_{\bar{q}} \delta_{T} w(x) \frac{2 \bar{u}(x)+2 \bar{d}(x)+s(x)+\bar{s}(x)}{6},(5)
\end{aligned}
$$

where flavor-symmetric tensor-polarized antiquark distributions are assumed. Tensor polarized heavy-quark distributions are neglected in this work. To be precise, the distributions extend to $x=2$ in the deuteron, whereas the kinematical limit is $x=1$ for the nucleon. Therefore, the tensor-polarized distributions given in Eq. (5) cannot describe the region at $1<x<2$. However, the PDFs are very small and it is not the stage to investigate the tensor distributions in such a large- $x$ region.

We analyze the data in the leading order (LO) of the running coupling constant $\alpha_{s}$. The structure function $b_{1}$ is then given by

$$
\begin{aligned}
b_{1}^{D}(x) & =\frac{1}{36} \delta_{T} w(x)\left[5\left\{u_{v}(x)+d_{v}(x)\right\}\right. \\
& \left.+4 \alpha_{\bar{q}}\{2 \bar{u}(x)+2 \bar{d}(x)+s(x)+\bar{s}(x)\}\right] .
\end{aligned}
$$

The unpolarized PDFs $u_{v}(x), d_{v}(x), \cdots, \bar{s}(x)$ could be taken from a recent global analysis, for example, by CTEQ [22], GJR 223], or MSTW [24]. In this work, the LO version of the MSTW parametrization is used. For the functional form of $\delta_{T} w(x)$, we note that there is a constraint from the sum rule in Eq. (3). The integrated tensor polarization, namely the first moment, should vanish for the valence quarks. It indicates that there should be a node in the $x$-dependent function, so that an appropriate parametrization could be

$$
\delta_{T} w(x)=a x^{b}(1-x)^{c}\left(x_{0}-x\right),
$$

where $x_{0}$ is the position where $\delta_{T} q_{v}(x)$ (and $\delta_{T} \bar{q}(x)$ ) vanishes. If the first moments vanish for the valence-quark distributions, the constant $x_{0}$ is expressed by the other parameters as

$$
x_{0}=\frac{\int_{0}^{1} d x x^{b+1}(1-x)^{c}\left\{u_{v}(x)+d_{v}(x)\right\}}{\int_{0}^{1} d x x^{b}(1-x)^{c}\left\{u_{v}(x)+d_{v}(x)\right\}} .
$$

The $a, b, c$, and $\alpha_{\bar{q}}$ are the parameters to be determined from experimental measurements.

The parametrization of Eq. (77) is motivated by the following considerations. First, the parton model indicates the existence of a node as mentioned. Next, we expect to have smooth polynomial functional forms in the limits, $x \rightarrow 0$ and 1 , as usual in unpolarized and longitudinallypolarized PDFs. In addition, the existence of the node and the functional form are, for example, supported by theoretical estimates of a convolution model, or so called binding model, where the $D$-state admixture gives rise to an $x$-distribution with a node in $b_{1}$ including the tensorpolarized antiquark distributions [5, 6, 9].

From an analysis of the HERMES experimental data, the optimum function $\delta_{T} w(x)$ and $\alpha_{\bar{q}}$ are determined. It is obvious from the data that the $b_{1}$ structure functions are not accurately measured to discuss scaling violation or even details of $x$ dependence. Therefore, a simplification is made by ignoring the scaling violation. The $Q^{2}$ value is fixed at $Q^{2}=2.5 \mathrm{GeV}^{2}$, which is about the average $Q^{2}$ of the HERMES measurements, for calculating 
TABLE I: Determined parameters in Eqs. (5) and (7). $Q^{2}$ is taken $Q^{2}=2.5 \mathrm{GeV}^{2}$.

\begin{tabular}{c|cccccc}
\hline Analysis & $\chi^{2} /$ d.o.f. & $a$ & $\alpha_{\bar{q}}$ & $b$ & $c$ & $x_{0}$ \\
\hline Set 1 & 2.83 & $0.378 \pm 0.212$ & 0.0 (fixed) & $0.706 \pm 0.324$ & 1.0 (fixed) & 0.229 \\
Set 2 & 1.57 & $0.221 \pm 0.174$ & $3.20 \pm 2.75$ & $0.648 \pm 0.342$ & 1.0 (fixed) & 0.221 \\
\hline
\end{tabular}

the unpolarized PDFs [24] in Eqs. (5) and (6). We made two types of analyses:

- Set 1: Tensor-polarized antiquark distributions are terminated $\left(\alpha_{\bar{q}}=0\right)$.

- Set 2: Finite tensor-polarized antiquark distributions are allowed $\left(\alpha_{\bar{q}}\right.$ is a parameter).

Due to the lack of data at large $x$, the parameter $c$ cannot be determined from the current data. We checked that the $\chi^{2}$ value is not much affected by this parameter. Therefore, it is fixed at $c=1$ in our analyses.

The determined parameters are listed in Table II It is obvious that the fit is not good enough $\left(\chi^{2} /\right.$ d.o.f. $\left.=2.83\right)$ if the tensor-polarized antiquark distributions are terminated (set 1 ) by fixing the parameter as $\alpha_{\bar{q}}=0$. If we let this parameter free in the analysis, it is a reasonably successful one $\left(\chi^{2} /\right.$ d.o.f. $\left.=1.57\right)$. It is interesting to find that the parameter $\alpha_{\bar{q}}$ is larger than one, which indicates that a significant tensor polarization exists in the antiquark distributions.

Analysis results are shown in Fig. 10 in comparison with the HERMES experimental data. Only the data with $Q^{2}>1 \mathrm{GeV}^{2}$ are included in the analyses. The set-1 curve is shown by the dashed curve, which does not agree with the data in the small- $x$ region $(x<0.1)$ without the antiquark polarization. The overall fit is successful only if the antiquark polarization is introduced (set 2) as shown by the solid curve. Of course, the results depend on the assumed functional form including the assumption of using the common weight function $\delta_{T} w(x)$ for the quark and antiquark distributions. However, it

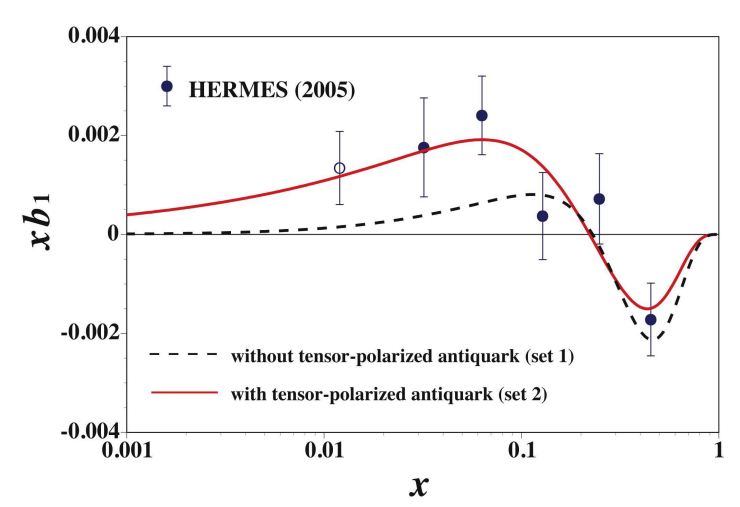

FIG. 1: (Color online) Comparison with HERMES data [19]. The solid and dashed curves indicate theoretical results with $\left(\alpha_{\bar{q}} \neq 0\right)$ and without $\left(\alpha_{\bar{q}}=0\right)$ tensor-polarized antiquark distributions. The open circle is the data at $Q^{2}<1 \mathrm{GeV}^{2}$. would be reasonable as long as a smooth $x$-dependence is valid for the weight function $\delta_{T} w(x)$.

The determined tensor-polarized distributions in Eq. (5) are shown in Fig. 2 by using the parameters in Table [I. The amplitude is sightly larger for the valence-quark distribution of set 1 because the antiquark distributions are terminated by setting $\alpha_{\bar{q}}=0$. The antiquark distribution $\delta_{T} \bar{q}^{D}$ is shown by the dotted curve and it is mainly distributed in the region $x<0.1$. It is interesting to find that a finite antiquark tensor polarization is needed to explain the HERMES data on $b_{1}$. If its effect on the $b_{1}$ sum rule is estimated, we obtain

$$
\begin{aligned}
\int d x b_{1}(x)=- & \frac{5}{24} \lim _{t \rightarrow 0} t F_{Q}(t)+\frac{1}{18} \int d x\left[8 \delta_{T} \bar{u}(x)\right. \\
& \left.+2 \delta_{T} \bar{d}(x)+\delta_{T} s(x)+\delta_{T} \bar{s}(x)\right] \\
= & 0.0058 .
\end{aligned}
$$

The choice of parametrization of Eq. (77) for the antiquark distributions could affect the numerical result. However, as it is obvious from Figs. 1 and 2, the antiquarks contribute only at small $x(x<0.1)$. As long as the function $\delta_{T} w(x)$ is a smooth function at $x<0.1$, the result is not significantly changed.

This work is the first attempt to parametrize the tensor polarized valence-quark and antiquark distributions. Including the antiquark tensor polarization, we obtained much smaller $\chi^{2} /$ d.o.f. and it led to a finite sum as shown in Eq. (9). This is a new and interesting result which needs to be explained theoretically. The integral is compared with the HERMES results [19], $\int_{0.002}^{0.85} d x b_{1}(x)=[1.05 \pm 0.34($ stat $) \pm 0.35($ sys $)] \times 10^{-2}$

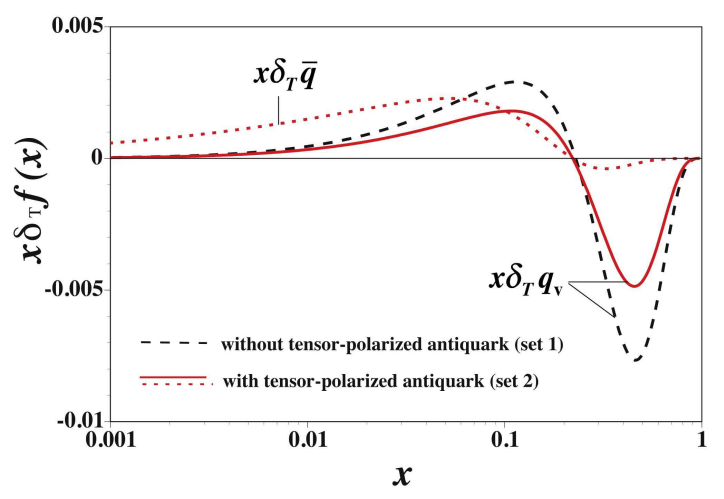

FIG. 2: (Color online) Determined tensor-polarized distributions. The dashed and solid curves are the valence-quark distributions $x \delta_{T} q_{v}^{D}$ in the deuteron for set $1\left(\alpha_{\bar{q}}=0\right)$ and set 2 $\left(\alpha_{\bar{q}} \neq 0\right)$, respectively, and the dotted curve is the antiquark distribution $x \delta_{T} \bar{q}^{D}$ of set 2 . 
and $\int_{0.02}^{0.85} d x b_{1}(x)=[0.35 \pm 0.10($ stat $) \pm 0.18($ sys $)] \times 10^{-2}$ in the restricted range with $Q^{2}>1 \mathrm{GeV}^{2}$. The integral of Eq. (9) is similar to the Gottfried sum [25]

$$
\int \frac{d x}{x}\left[F_{2}^{p}(x)-F_{2}^{n}(x)\right]=\frac{1}{3}+\frac{2}{3} \int[\bar{u}(x)-\bar{d}(x)],
$$

where the deviation from $\int\left[u_{v}(x)-d_{v}(x)\right] / 3=1 / 3$ indicates flavor asymmetric antiquark distributions. In the $b_{1}$ case, the finite sum $\int d x b_{1}$ suggests that a finite tensorpolarized antiquark distribution should exist.

It is obvious from Fig. 1 that much better measurements are needed to investigate the details of tensorpolarized distributions particularly at medium and large $x$ (>0.2). Such measurements could be possible, for example, at JLab (Thomas Jefferson National Accelerator Facility) by measuring $b_{1}$ and also at hadron facilities such as J-PARC (Japan Proton Accelerator Research Complex) 26] GSI-FAIR (Gesellschaft für Schwerionenforschung -Facility for Antiproton and Ion Research) [27] by Drell-Yan processes with polarized deuteron [12]. In particular, the Drell-Yan processes are suitable for di- rectly finding the tensor-polarized antiquark distributions in Eq. (9).

Summary: In this work, optimum tensor-polarized quark and antiquark distributions are proposed from the analyses of HERMES data on $b_{1}$ for the deuteron. We found that a significant antiquark tensor polarization exists if the overall tensor polarization vanishes for the valence quarks although such a result could depend on the assumed functional form. Further experimental measurements are needed for $b_{1}$ such as at JLab as well as Drell-Yan measurements with tensor-polarized deuteron at hadron facilities, J-PARC and GSI-FAIR. On the other hand, it is interesting to conjecture a possible physics mechanism to create a finite tensor-polarized antiquark distribution.

\section{Acknowledgments}

The author thanks C. Ciofi degli Atti, T.-Y. Kimura, Y. Miyachi, and O. V. Teryaev for discussions on structure functions of spin-one hadrons.
[1] J. Ashman et al. (European Muon Collaboration), Phys. Lett. B 206, 364 (1988).

[2] J. Blümlein and H. Böttcher, arXiv:1005.3113 [hep-ph]; D. de Florian, R. Sassot, M. Stratmann, and W. Vogelsang, Phys. Rev. D 80, 034030 (2009). S. E. Kuhn, J.-P. Chen, and E. Leader, Prog. Part .Nucl. Phys. 63, 1 (2009); M. Hirai and S. Kumano, Nucl. Phys. B 813, 106 (2009).

[3] Recent studies are explained in A. Airapetian et al. (HERMES Collaboration), arXiv:1004.0177 [hep-ex]; M. Guidal, Prog. Part. Nucl. Phys. 61, 89 (2008); C. Weiss, AIP Conf. Proc. 1149, 150 (2009).

[4] S. Kumano, M. Strikman, and K. Sudoh, Phys. Rev. D 80, 074003 (2009).

[5] L. L. Frankfurt and M. I. Strikman, Nucl. Phys. A 405, 557 (1983).

[6] P. Hoodbhoy, R. L. Jaffe, and A. Manohar, Nucl. Phys. B 312, 571 (1989).

[7] R. L. Jaffe and A. Manohar, Nucl. Phys. B 321, 343 (1989). B. Lampe and E. Reya, Phys. Rept. 332, 1 (2000); A. Bacchetta and P. J. Mulders, Phys. Rev. D 62, 114004 (2000).

[8] F. E. Close and S. Kumano, Phys. Rev. D 42, 2377 (1990).

[9] H. Khan and P. Hoodbhoy, Phys. Rev. C 44, 1219 (1991).

[10] G. A. Miller, pp.30-33 in Topical Conference on Electronuclear physics with Internal Targets, edited by R. G. Arnold (World Scientific, Singapore, 1990).

[11] For example, see N. N. Nikolaev and W. Schäfer, Phys. Lett. B 398, 245 (1997); Erratum, ibid., B 407, 453 (1997); K. Bora and R. L. Jaffe, Phys. Rev. D 57, 6906 (1998).

[12] S. Hino and S. Kumano, Phys. Rev. D 59, 094026 (1999); 60, 054018 (1999); S. Kumano and M. Miyama, Phys.
Lett. B 479, 149 (2000).

[13] A. Schäfer, L. Szymanowski, and O. V. Teryaev, Phys. Lett. B 464, 94 (1999).

[14] E. R. Berger, F. Cano, M. Diehl, and B. Pire, Phys. Rev. Lett. 87, 142302 (2001); A. Kirchner and D. Mueller, Eur. Phys. J. C 32, 347 (2003); M. Diehl, Phys. Rept. 388, 41 (2003); F. Cano and B. Pire, Eur. Phys. J. A 19, 423 (2004); A. V. Belitsky and A. V. Radyushkin, Phys. Rept. 418, 1 (2005).

[15] W. Detmold, Phys. Lett. B 632, 261 (2006).

[16] V. Dmitrasinovic, Phys. Rev. D 54, 1237 (1996).

[17] C. Best et al., Phys. Rev. D 56, 2743 (1997).

[18] T.-Y. Kimura and S. Kumano, Phys. Rev. D 78, 117505 (2008).

[19] A. Airapetian et al. (HERMES Collaboration), Phys. Rev. Lett. 95, 242001 (2005).

[20] The overall factor $1 / 2$ is introduced in $b_{1}$ as usual in defining $F_{1}$ and $g_{1}$ in terms of PDFs.

[21] M. Hirai, S. Kumano, and T.-H. Nagai, Phys. Rev. C 76, 065207 (2007) and references therein.

[22] P. M. Nadolsky et al., Phys. Rev. D 78, 013004 (2008).

[23] M. Glc̈k, P. Jimenez-Delgado, and E. Reya, Eur. Phys. J. C 53, 355 (2008).

[24] A. D. Martin, W. J. Stirling, R. S. Thorne, and G. Watt, Eur. Phys. J. C 63, 189 (2009). The LO PDFs are used in this work.

[25] S. Kumano, Phys. Rept. 303, 183 (1998); G. T. Garvey and J.-C. Peng, Prog. Part. Nucl. Phys. 47, 203 (2001).

[26] See http://j-parc.jp/index-e.html for the J-PARC project. S. Kumano, Nucl. Phys. A 782, 442 (2007); AIP Conf. Proc. 1056, 444 (2008).

[27] http://www.gsi.de/fair/index_e.html. 